- a indução da expressão e síntese de outros fatores profibrogénicos, tais como a vimentina e o CTGF;

\title{
$\checkmark$ Losartan no Tratamento das Lesões Musculares
}

\section{Dr. Filipe Cabral ${ }^{1}$, Prof. Doutor Pedro Barata ${ }^{2}$}

${ }^{1}$ Interno de Formação Específica de Medicina Geral e Familiar, Pós-Graduado em Medicina Desportiva; USF Marco - ACeS Tâmega I - Baixo Tâmega, Marco de Canaveses; ${ }^{2}$ Pós-Graduado em Medicina Desportiva; Faculdade de Ciências da Saúde da Universidade Fernando Pessoa, I3S-UP, Centro Hospitalar Trás-os-Montes e Alto Douro.

\begin{abstract}
RESUMO / ABSTRACT
As lesões musculares são bastante prevalentes na prática desportiva. Frequentemente o processo de reparação muscular culmina na formação de uma cicatriz fibrótica, a qual não só limita a recuperação funcional completa, como também aumenta consideravelmente a probabilidade de recidiva. O TGF- $\beta 1$ constitui o principal fator profibrogénico envolvido neste processo cicatricial. Ao bloquear a sua atividade, o Losartan demonstrou eficácia na redução da fibrose e no aumento da capacidade regenerativa e funcional após lesão muscular, sendo por isso uma alternativa terapêutica a ser equacionada neste tipo de lesões.
\end{abstract}

\begin{abstract}
Muscle injuries are very common in sports medicine. Frequently the muscle repair process ends in the formation of a fibrotic scar, that not only limits the complete functional recovery, but also increases the likelihood of injury recurrence. TGF- $\beta 1$ is the main profibrogenic factor involved in this healing process. By blocking its activity, Losartan has proven it efficacy in reducing fibrosis and increasing regenerative and functional capacity post muscle injury. Therefore, its use should be considered as an alternative therapeutic for this kind of injuries.
\end{abstract}

PALAVRAS-CHAVE / KEYWORDS

Lesão muscular, fibrose, TGF- $\beta 1$, losartan

Muscle injury, fibrosis, TGF- $\beta 1$, losartan

\section{Introdução}

As lesões musculares são bastante comuns na prática desportiva ${ }^{1,2}$, constituindo cerca de $31 \%$ de todas as lesões verificadas no futebol de elite..$^{3,4}$ Além de bastante prevalentes são também uma causa importante de dias de ausência da prática desportiva, com todos os impactos no desempenho estratégico, competitivo e financeiro daí inerentes. ${ }^{2-4} \mathrm{Em}$ atletas de alta competição, a taxa de recidiva, embora varie consoante o grupo muscular e área envolvida, pode chegar aos 30\%. ${ }^{5,6}$ Assim, a otimização do processo preventivo, diagnóstico, terapêutico e de reabilitação deste tipo de lesões constitui uma área de grande interesse na atualidade e em torno da qual se efetua numerosa investigação científica.

Os mecanismos de reparação muscular após a lesão envolvem processos sequenciais complexos e interdependentes entre si cujo objetivo final consiste na restauração da arquitetura e da funcionalidade tecidular prévia. ${ }^{5}$ Todavia, esta capacidade é limitada e frequentemente culmina na formação de uma cicatriz fibrótica, a qual não só atrasa/ limita a recuperação funcional completa, como também aumenta a suscetibilidade de recorrência. ${ }^{5-9}$ A compreensão dos mecanismos fisiopatológicos envolvidos neste processo cicatricial tem sido alvo de vários estudos, no sentido de identificar potenciais alvos terapêuticos que promovam a redução do processo cicatricial, com todos os potenciais benefícios que daí possam advir.

Múltiplos fatores de crescimento, tais como o Epidermal Growth Factor, o Fibroblast Growth Factor e o Connective Tissue Growth Factor (CTGF), estão envolvidos no processo cicatricial fibrótico. Todavia, o Transforming Growth Factor Beta 1 (TGF- $\beta 1$ ) é aceite na literatura como sendo o principal agente responsável pela sua formação. ${ }^{6,8-12}$ Os mecanismos a partir dos quais o TGF- $\beta 1$ promove a ocorrência de fibrose tecidular são bastante variados e complexos, destacando-se:6-9,11,13

- a alteração do equilíbrio entre a síntese e a degradação da matriz extracelular;
- o aumento da sua própria expressão tecidular ao longo de toda a zona lesada através de sinais autócrinos (processo secundário à ativação da via dependente do Smad2/3). ${ }^{6}$

O TGF- $\beta 1$ tem ainda amplos efeitos no crescimento, diferenciação e modulação da resposta imune local, inibindo a diferenciação das células satélite durante o processo regenerativo, dificultando, deste modo, de forma sinérgica, todo o processo de recuperação após lesão. 5,7,9-11,13 Por conseguinte, agentes que potenciem a neutralização e/ou a down-regulation do TGF- $\beta 1$ parecem ser a melhor estratégia para reduzir a formação deste processo cicatricial fibrótico.

Vários estudos demonstraram um aumento da expressão dos recetores da angiotensina II na matriz extracelular da zona lesionada, sugerindo que a up-regulation destes mesmos recetores estaria intimamente relacionada com a deposição de tecido fibrótico e que o aumento dos níveis de TGF- $\beta 1$ locais seria resultado potencial da atividade da angiotensina II (à semelhança do que acontece noutros tecidos do organismo). ${ }^{2,5-7,10,12,13}$ Nesse contexto, a utilização de agentes que inibam/ diminuam a atividade da angiotensina II, como é o caso do losartan, levam a potencial redução da expressão e da atividade do TGF- $\beta 1$ e, subsequentemente, a diminuição do processo de fibrose pós-lesional, aumento da capacidade de regeneração muscular, diminuição do risco de recidiva e promoção do retorno à competição mais rápido..,6

O losartan foi o primeiro antagonista oral do recetor tipo 1 da angiotensina II introduzido no mercado, sendo utilizado frequentemente no tratamento da hipertensão arterial, quer em monoterapia, quer em associação. ${ }^{7,9}$ Mais recentemente, após a descoberta da correlação entre o aumento dos níveis de losartan e o aumento da produção de TGF- $\beta 1$, emergiu como potente terapêutica antifibrótica. ${ }^{6,7,9,10}$ Os seus efeitos antifibróticos estão relacionados não só com a inibição da atividade, do CTGF, mas particularmente com a inibição do TGF- $\beta 1 . .^{5,6-8,11}$ Esta ação 
ocorre através da inibição direta do recetor do tipo 1 da angiotensina II, com consequente inibição da via dependente do Smad2/36-8,11, à semelhança com o que ocorre em muitas outras patologias fibróticas (fibrose pulmonar, doença renal, cardiomiopatias, aneurismas da aorta). 2,5-7,10-13 Porém, ao contrário do verificado nestas últimas, a evidência da utilização do losartan como terapêutica promotora da diminuição do processo de fibrose e melhoria da regeneração e funcionalidade muscular após uma lesão muscular esquelética é, ainda, escassa.

Neste sentido, o principal objetivo deste trabalho consistiu em rever qual a evidência existente acerca da utilização do losartan no tratamento das lesões musculares.

\section{Métodos}

Foi realizada uma pesquisa de normas de orientação clínica, meta-análises, revisões sistemáticas e ensaios clínicos na MEDLINE e nas bases de dados National Guideline Clearinghouse, National Electronic Library for Health do National Health Service britânico, Canadian Medical Association Practice Guidelines Infobase, The Cochrane Library, DARE e Bandolier, a 1 de maio de 2020, utilizando os termos MeSH "Losartan" e "Muscles/ injuries”. Além disso, foram procurados ensaios clínicos concluídos e em curso nas plataformas clinicaltrials.gov e EudraCT. Foram incluídos artigos escritos na língua inglesa, francesa, espanhola e portuguesa, publicados nos últimos 15 anos e cujo objetivo principal consistia em avaliar o papel do losartan no tratamento das lesões musculares, quer em monoterapia, quer em associação com outras substâncias/terapias. Foram excluídos artigos que não cumpriam os critérios de inclusão, artigos duplicados ou presentes noutras meta-análises e/ou revisões sistemáticas e artigos de opinião.

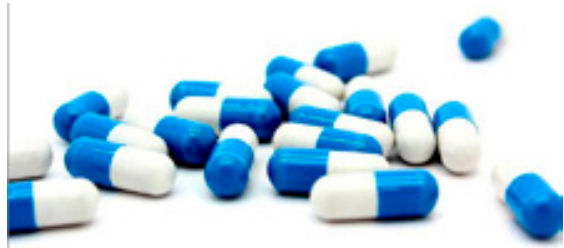

https://akm-img-a-in.tosshub.com/sites/btmt/images/ stories/pills_660_180220033548.jpg

\section{Resultados}

Foram encontrados oito artigos que correspondiam aos critérios estabelecidos e que, por conseguinte, foram incluídos nesta revisão. Todos eles demonstraram que a terapêutica com losartan diminuía significativamente o processo de fibrose tecidual após lesão muscular, sendo esse fenómeno à custa da inibição do TGF- $\beta 1 .{ }^{2,6-12}$ Adicionalmente, ao aumentar o número de fibras musculares regenerativas (centro-nucleadas) na zona da lesão, a utilização de losartan aumenta a capacidade regenerativa local, sendo este, muito provavelmente, um mecanismo dose-dependente. 2,5,6 Estes dois processos, aliados à capacidade aumentada de fusão e proliferação dos mioblastos, poderão explicar a potenciação funcional e da força muscular encontrada aquando da sua utilização. 2,5,7

Quando avaliada a terapêutica combinada de losartan com fatores de crescimento ou células estaminais, verificou-se a melhoria da capacidade de cicatrização e regeneração muscular superior à verificada aquando da utilização destas terapêuticas isoladamente. , $8,10,12^{2}$ Satoshi Terada et al. ${ }^{7}$ desenvolveram um estudo animal que pretendeu explorar o efeito do tratamento com plasma rico em plaquetas (PRPs), losartan e sua combinação nas contusões musculares. Para tal avaliaram os efeitos da administração injetável de 20mL de PRPs um dia após um episódio de contusão muscular us administração de 10mg/ kg/dia de losartan três dias após lesão us administração combinada destas duas substâncias us placebo. Através da análise histológica local e aplicação de testes fisiológicos, os autores constataram que, além dos benefícios já supracitados, a utilização destes dois compostos em associação tem um efeito sinérgico na promoção e no aumento da capacidade de revascularização muscular (secundário ao aumento da expressão de Vascular Endothelial Growth Factor) e, paralelamente, na inibição do processo fibrótico, o que em última instância leva ao aumento da capacidade funcional e, subsequente, da melhoria da força muscular. Johnny Huard et al. ${ }^{12}$, além de corroborarem estes achados, salientam ainda na sua revisão, que a utilização combinada de PRPs com losartan, em doses equiparadas aos do estudo supracitado, está associada ainda a melhoria na capacidade de reparação cartilagínea pós-operatória. Jin-Kyu Park et al. ${ }^{10}$ verificaram a ocorrência deste mesmo efeito sinérgico, neste caso com a utilização combinada de células estaminais derivadas de tecido adiposo (ASCs) e losartan - injeção de $5 \times 10^{\wedge} 5$ ASCs no dia 1 pós laceração muscular e losartan ad libitum durante duas semanas juntamente com água numa concentração de 0.6g/L). Para averiguar qual o papel do losartan em associação com células estaminais derivadas do tecido muscular (MDSCs) nas contusões musculares, Makoto Kobayashi et al. ${ }^{8}$ desenharam um estudo no qual compararam os efeitos da administração de $3 \times 10^{\wedge} 5$ MDSCs quatro dias após lesão us a administração desse mesmo composto em associação com 10mg/ $\mathrm{kg} /$ dia de losartan oral a partir do 3. dia pós-lesional. Mais uma vez, através da análise histológica local e de testes fisiológicos (realizados duas e quatro semanas após lesão), os autores constataram diminuição significativa do processo cicatricial fibrótico, aumento do número de fibras musculares regenerativas e aumento da capacidade funcional superior ao verificado aquando da administração destas substâncias em forma isolada.

A maioria dos estudos salienta ainda o excelente perfil de segurança e eficácia associado ao uso do losartan, devendo a sua utilização, por conseguinte, ser equacionada no tratamento não-cirúrgico das lesões musculares esqueléticas. ${ }^{2,5-7,10}$

\section{Discussão}

As lesões musculares constituem cerca de $1 / 3$ de todas as lesões verificadas no futebol, sendo uma causa frequente de inatividade física. O processo de reparação muscular é bastante complexo e, não raramente, resulta na formação de uma cicatriz fibrótica. De todos os fatores de crescimento envolvidos neste processo cicatricial, o TGF- $\beta 1$ constitui o principal agente profibrogénico responsável pela sua 
formação. O losartan, ao bloquear a ação do TGF- $\beta 1$, revelou de forma consistente ser eficaz na redução da fibrose tecidular e na melhoria da capacidade regenerativa e funcional muscular após uma lesão muscular.

Esta revisão apresenta, contudo, algumas limitações. De destacar, em primeiro lugar, que embora existam diferentes tipos de mecanismos lesionais e, subsequentemente, diferentes tipos de lesões musculares, as publicações incluídas avaliaram o papel do losartan no tratamento de lesões secundárias a processos de contusão e/ou laceração, não sendo, por isso, possível inferir as mesmas conclusões noutro tipo de lesões musculares. Adicionalmente, sete dos oito estudos incluídos nesta revisão apresentam resultados in vitro e/ou em modelos animais, pelo que a sua aplicabilidade, segurança e eficácia em humanos carece de evidência científica mais robusta. Todavia, como pontos fortes, de destacar que o uso de losartan demonstrou, de forma consistente e em todas as publicações incluídas nesta revisão, ser uma alternativa terapêutica segura, eficaz, bem tolerada e que, como tal, a ser equacionada no tratamento não-cirúrgico das lesões musculares esqueléticas. Além disso, a sua utilização em combinação com fatores de crescimento ou células estaminais (como PRPs, ASCs e MDSCs), promove um efeito sinérgico no processo de cicatrização, regeneração e recuperação muscular após lesão com potenciais efeitos benefícios adicionais. ${ }^{7,8,10,12}$

Ensaios mais robustos serão necessários para aferir qual a dosagem, posologia e timing mais indicado para a utilização off-label do losartan no tratamento de lesões musculares em humanos. A maioria dos estudos que abordaram esta temática, sugerem a dose de 10mg/ kg/dia utilizada em modelo animal (o que equivale à dose diária utilizada no tratamento da hipertensão em humanos - 50 a 100mg/dia), ${ }^{2}$ instituída a partir do 3. dia após a lesão, como a melhor estratégia para aumentar a capacidade de regeneração e cicatrização muscular e de diminuir a fibrose tecidular. ${ }^{2,7-9}$

\section{Conclusão}

Em suma, a utilização de losartan demonstrou, via inibição do TGF- $\beta 1$, reduzir significativamente o processo de fibrose tecidual e aumentar a capacidade regenerativa local após uma lesão muscular. Estes fenómenos, em conjunto, associaram-se a uma melhoria da capacidade funcional e da força muscular. A utilização combinada de fatores de crescimento ou células estaminais com losartan demonstrou ter um efeito sinérgico e favorável neste processo de cicatrização, reparação e regeneração tecidular com potenciais benefícios adicionais. Novos estudos são imprescindíveis para que se possa aferir qual a dosagem, posologia e timing mais indicados para a utilização do losartan neste contexto, bem como para averiguar qual o papel desta molécula noutras matérias, tais como no tempo de retorno à competição após lesão e na taxa de recorrência das mesmas.

\section{Conflitos de Interesse}

Os autores declaram não haver conflitos de interesse que poderiam constituir um impedimento para a publicação deste artigo, assim como a sua originalidade e a não publicação prévia.

\section{Contacto}

Nome completo: Filipe Emanuel O. Cabral E-mail: f1cabral@hotmail.com

Telemóvel: +351938139594

Morada: Avenida 20 de Maio, n. ${ }^{\circ} 18,3 .{ }^{\circ}$ Centro-Traseiras, 4590-182 Paços de Ferreira

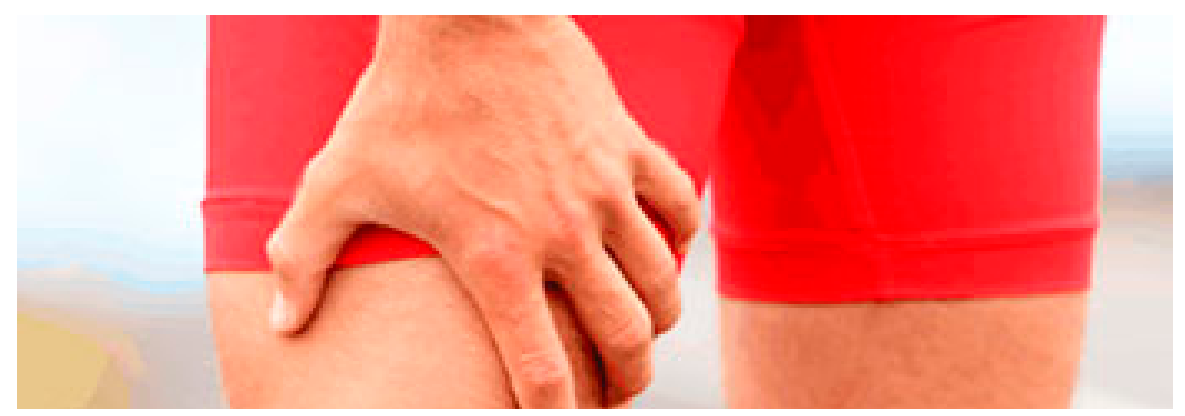

https://adrianoleonardi.com.br/artigos/lesao-muscular-posterior-da-coxa

1. Wohlfahrt H-W, Haensel L, Mithoefer K, et al. Terminology and classification of muscle injuries in sport: the munich consensus statement. Br J Sports Med. 2013; 47:342-350.

2. Kobayashi T, Uehara K, Ota S, Tobita K, Ambrosio F, Cummins JH, Terada S, Fu FH, Huard J. The timing of administration of a clinically relevant dose of losartan influences the healing process after contusion induced muscle injury. J Appl Physiol. 2013; 114:262-273.

3. Ekstrand J, Hagglund M, Walden M. Epidemiology of muscle injuries in professional football (soccer). Am J Sports Med 2011; 39:1226-32.

4. Ekstrand, J. Muscle Epidemiology and Injury Mechanisms. Encyclopedia of Footbal Medicine, Vol. II, Thieme, 2017; 22-30.

5. Gharaibeh B, Chun-Lansinger Y, Hagen T, Ingham SJM, Wright V, Fu F, Huard J. Biological approaches to improve skeletal muscle healing after injury and disease. Birth Defects Res C Embryo Today. 2012; 96(1):82-94.

6. Bedair HS, Karthikeyan T, Quintero A, Li Y, Huard J. Angiotensin II receptor blockade administered after injury improves muscle regeneration and decreases fibrosis in normal skeletal muscle. Am J Sports Med. 2008; 36(8):1548-54

7. Terada S, Ota S, Kobayashi M, Kobayashi T, Mifune Y, Takayama K, Witt M, Vadala G, Oyster N, Otsuka T, Fu FH, Huard J. Use of an antifibrotic agent improves the effect of platelet-rich plasma on muscle healing after injury. J Bone Joint Surg Am. 2013; 95:980-988.

8. Kobayashi M, Ota S, Terada S, Kawakami Y, Otsuka T, Fu FH, Huard J. The combined use of losartan and muscle-derived stem cells significantly improves the functional recovery of muscle in a young mouse model of contusion injuries. Am J Sports Med. 2016; 44:3252-61.

9. Garg K, Corona BT, Walters TJ. Losartan administration reduces fibrosis but hinders functional recovery after volumetric muscle loss injury. J Appl Physiol. 2014 117(10):1120-1131.

10. Park JK, Ki MR, Lee EM, Kim AY, You SY, Han SY, Lee EJ, Hong IH, Kwon SH, Kim SJ, Rando TA, Jeong KS. Losartan improves adipose tissue-derived stem cell niche by inhibiting transforming growth factor-beta and fibrosis in skeletal muscle injury. Cell Transplantation. 2012; 21:2407-2424.

11. Hwang OK, Park JK, Lee EJ, Lee EM, Kim AY, Jeong KS. Therapeutic effect of losartan, an angiotensin II type 1 receptor antagonist, on $\mathrm{CCl}^{4}$-induced skeletal muscle injury. Int J Mol Sci. 2016;8; 17(2):227.

12. Huard J, Bolia I, Briggs K, Utsunomiya H, Lowe WR, Philippon MJ. Potential usefulness of losartan as an antifibrotic agent and adjunct to platelet-rich plasma therapy to improve muscle healing and cartilage repair and prevent adhesion formation. Orthopedics. 2018; 41(5):e591-e597.

13. MacDonald EM, Cohn RD. TGF $\beta$ signaling: its role in fibrosis formation and myopathies. Curr Opin Rheumatol. 2012; 24(6):628-634. 